ARTICLE

\title{
A panel of human neutralizing $m A$ bs targeting SARS-CoV-2 spike at multiple epitopes
}

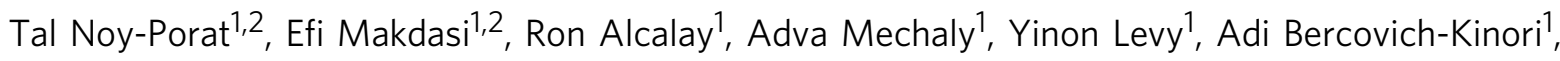
Ayelet Zauberman', Hadas Tamir', Yfat Yahalom-Ronen', Ma'ayan Israeli', Eyal Epstein', Hagit Achdout', Sharon Melamed ${ }^{1}$, Theodor Chitlaru', Shay Weiss ${ }^{1}$, Eldar Peretz ${ }^{1}$, Osnat Rosen ${ }^{1}$, Nir Paran ${ }^{1}$, Shmuel Yitzhaki ${ }^{1}$, Shmuel C. Shapira ${ }^{1}$, Tomer Israely ${ }^{1}$, Ohad Mazor ${ }^{1,3 凶} \&$ Ronit Rosenfeld ${ }^{1,3 凶}$

The novel highly transmissible human coronavirus SARS-CoV-2 is the causative agent of the COVID-19 pandemic. Thus far, there is no approved therapeutic drug specifically targeting this emerging virus. Here we report the isolation and characterization of a panel of human neutralizing monoclonal antibodies targeting the SARS-CoV-2 receptor binding domain (RBD). These antibodies were selected from a phage display library constructed using peripheral circulatory lymphocytes collected from patients at the acute phase of the disease. These neutralizing antibodies are shown to recognize distinct epitopes on the viral spike RBD. A subset of the antibodies exert their inhibitory activity by abrogating binding of the RBD to the human ACE2 receptor. The human monoclonal antibodies described here represent a promising basis for the design of efficient combined post-exposure therapy for SARS-CoV-2 infection.

\footnotetext{
${ }^{1}$ Israel Institute for Biological Research, Ness-Ziona, Israel. ${ }^{2}$ These authors contributed equally: Tal Noy-Porat, Efi Makdasi. ${ }^{3}$ These authors jointly supervised this work: Ohad Mazor and Ronit Rosenfeld. ${ }^{凶}$ email: ohadm@iibr.gov.il; ronitr@iibr.gov.il
} 
T he present global pandemic of coronavirus induced disease 19 (COVID-19), declared by the World Health Organization (WHO) as a public health emergency of international concern, is caused by the highly transmissible severe acute respiratory syndrome coronavirus 2 (SARS-CoV-2). To this date (end of June, 2020), about 10 million confirmed cases and 500,000 deaths have been reported worldwide ${ }^{1}$. Yet, there is no approved therapeutic drug specifically targeting the SARS-CoV-2. The novel coronavirus SARS-CoV-2, emerged as the seventh type of coronavirus infecting humans and the third most pathogenic preceded by the Severe Acute Respiratory Syndrome coronavirus (SARS-CoV) and the Middle East Respiratory Syndrome coronavirus (MERS-CoV).

Due to their exceptional antigen specificity, therapeutic monoclonal antibodies (mAbs) are considered an attractive candidate to target exposed antigenic sites on viruses, and prevent their infectivity ${ }^{2}$. While the therapeutic potential of mAbs, specifically targeting surface viral proteins was demonstrated for SARS-CoV ${ }^{3}$ and $\mathrm{MERS}^{4,5}$, only antibodies specific for Ebola are currently undergoing the more advanced clinical studies ${ }^{6}$.

SARS-CoV-2 utilizes the surface homotrimeric spike glycoprotein (S) as a major mediator of cellular infection ${ }^{7,8}$. The SARS$\mathrm{CoV}-2 \mathrm{~S}$ protein is composed of two distinct subunits, $\mathrm{S} 1$ and S2. The S1 subunit contains the receptor-binding domain (RBD), known to bind the Angiotensin-Converting Enzyme 2 (ACE2) receptor on host cell surfaces and the S2 subunit mediates the fusion of the viral and cellular membranes, essential for viral entry into the cell ${ }^{8,9}$. The receptor interaction site on S1 is considered the main target for efficient neutralization of cell infection and therefore a prime candidate for therapeutic antibody development $\mathrm{t}^{10-14}$. Although the $\mathrm{S}$ protein of the SARS-CoV and SARS-CoV-2 share $77.5 \%$ identity, most of the mAbs isolated against SARS-CoV reportedly failed to cross-neutralize the SARSCoV-2 virus ${ }^{15-17}$.

For designing optimal therapeutic strategies, there is an urgent need for the identification of neutralizing monoclonal antibodies that specifically target SARS-CoV-2. Such antibodies may be identified either in patients in the course of illness/recovery, or in immunized animals. In addition, alternative antibody discovery strategies may be applied, e.g., using synthetic $\mathrm{Ab}$ libraries. Recently, first two mAbs, elicited against the SARS-CoV-2, were reported ${ }^{18}$. Furthermore, efficient post-exposure therapy in humans, may require integration of several noncompeting mAbs, ideally neutralizing the virus infectivity by different mechanisms. Such combined therapies are expected to provide superior control of potential neutralizing escape variants ${ }^{11,19}$.

Here we describe the isolation of a panel of neutralizing $\mathrm{mAbs}$ selected against the SARS-CoV-2 RBD from phage display library constructed based on patient samples collected in the acute phase of the disease. These specific antibodies were found to recognize distinct epitopes and can potentially be used either for therapy or immune prophylaxis.

\section{Results and discussion}

Isolation of anti-SARS-CoV-2 antibodies. Several blood samples, either from COVID-19 convalescence or from patients with severe ongoing disease, were evaluated for titers of RBD binding and viral neutralization activity. Two blood samples derived from patients at the acute phase of disease, exhibiting the highest neutralizing ability $\left(\mathrm{NT}_{50}>5000\right)$ and demonstrating significant binding to both S1 subunit [DIL 50 (half-dilution value) of 494 and 473] and RBD (DIL 50 of 252 and 226; Fig. 1a), were subsequently selected for antibody library generation.

A phage display (PD) single-chain $\mathrm{Fv}(\mathrm{scFv})$ library, representing approx. 10 million distinct antibodies, was constructed. With the objective of isolating neutralizing Abs, three consecutive enrichment steps of panning were performed against both $\mathrm{mFc}$ S1 and huFc-RBD. Clones resulting from these enrichment steps, were tested by ELISA for their ability to bind huFc-RBD or huFcNTD. RBD-specific binders resulting from this screen were expressed as full-length antibodies (in a scFv-Fc format) for further analysis. Subsequently, eight RBD-specific antibodies carrying unique sequences were selected (Fig. 1b; Supplementary Fig. 1). Of note, no clones exhibiting anti NTD specificity were detected by the ELISA of those resulting from enrichment of the library against the S1 subunit. Furthermore, the clones resulting from enrichment using the full-length S1 subunit, were identical to those obtained following enrichment against RBD only.

Binding assays of these eight antibodies confirmed their specificity to the spike protein, the S1 subunit, and the RBD, while no reactivity was observed against the spike $\mathrm{N}$ terminal domain (NTD) of the spike protein (Fig. 1c). Evaluation of the Abs affinity toward S1 by ELISA evidenced apparent $\mathrm{K}_{\mathrm{D}}$ values of $1.8-3.8 \mathrm{nM}$ of six of the antibodies, and a $\mathrm{K}_{\mathrm{D}}$ values of 12.7 and $15.6 \mathrm{nM}$ of MD45 and MD63, respectively (Fig. 1d). To further characterize these antibodies, biolayer interferometry (BLI) measurements of the antibodies affinity specifically toward RBD was conducted, revealing $\mathrm{K}_{\mathrm{D}}$ values of $0.4-5.8 \mathrm{nM}$ for all antibodies, with MD29 showing the highest affinity and MD67 showing the lowest affinity (Fig. 1e; Supplementary Fig. 2).

Additional sequence analysis by IgBlast ${ }^{20}$ (Fig. 1b) revealed that antibodies MD17, MD29, and MD63 share common germ line origin of both their VH and VK (IGHV3-64 and IGKV1-39, respectively). Similarly, MD45 and MD67, share common VH and VK germ lines (IGHV3-53 and IGKV3-20). The VH of MD62, is similar to the one of MD45 and MD67, accompanied by a unique VK (IGKV1-12), while MD65 shared the same VK with MD45 and MD67, accompanied by a unique VH (IGHV3-66). MD47 originated from unique VH (IGHV3-23) and was the only $\mathrm{mAb}$, carrying VL (IGLV3-21).

Classification of antibody epitopes. SARS-CoV-2 spike RBD is known to mediate the binding of the human ACE2 receptor and thus, this domain is considered as main target for neutralizing mAbs. However, direct blocking of the RBD-ACE2 interaction is not the exclusive modality by which neutralizing antibodies can exert their effect ${ }^{21}$. Consequently, the selected mAbs, were classified on the basis of their epitope specificity determined by BLI epitope binning. In this assay, each individual antibody was biotinylated, immobilized to a streptavidin sensor, loaded with RBD and then challenged with each of the other antibodies. Simultaneous binding of the second antibody to RBD induces a wavelength shift in the interference pattern, which indicates that the two antibodies bind to non-overlapping epitopes ${ }^{21}$. Conversely, if the two antibodies bind the same or partiallyoverlapping epitope on RBD, no or very low wavelength shift, respectively, is induced. As a representative example, sensograms of the various antibody interactions with a pre-complexed MD65RBD is shown (Fig. 2a). Antibody MD65 used as a negative control, and did not elicit any wavelength shift, as expected. In contrast, antibodies MD29, MD47, MD62, and MD63 induced a marked wavelength shift indicating that they could bind to RBD simultaneously with MD65. The analysis revealed that antibodies MD45 and MD67 could not bind to RBD in the presence of MD65, indicating that these three antibodies target the same epitope. Analysis was then performed for the next seven antibodies (Supplementary Figs. 3 and 4 ) and the ability of each pair to simultaneously bind RBD was determined (Fig. 2b). Results ranged from full to no competition and enabled the classification of the mAbs into four groups recognizing distinct epitopes 
a

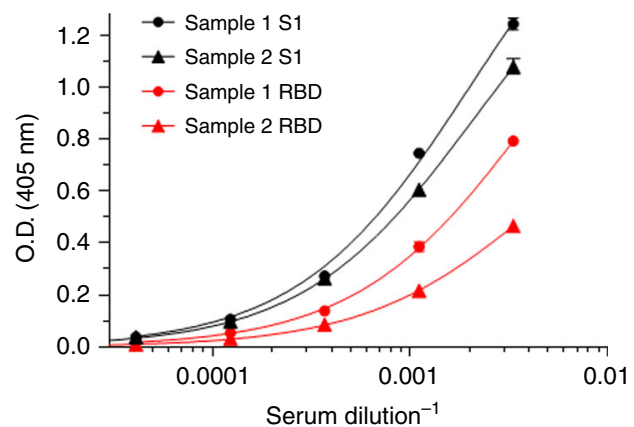

b

\begin{tabular}{|c|c|c|c|c|c|}
\hline \multirow[b]{3}{*}{ MD17 } & \multicolumn{2}{|l|}{ Heavy Chain } & \multicolumn{3}{|c|}{ LIGHT Chain } \\
\hline & HCDR3 & Germline & LCDR3 & & Germline \\
\hline & CVK DQDSSSWYDAFDI WG & IGHV3-64 & TYYC QQSYTTPLT & FGG & IGKV1-39 \\
\hline MD29 & CVK DQDSSSWYDAFDI WC & IGHV3-64 & ATYC HQTYTSPYT & $\mathrm{FGO}$ & IGKV1-39 \\
\hline MD63 & CVK DQDSNSWYDAFDI WC & IGHV3-64 & TYYC QQSYSTPYT & FGQ & IGKV1-39 \\
\hline MD65 & CAR DLAVAGAFDI & IGHV3-66 & WFC QQYGSSPLT & FGG & IGKV3-20 \\
\hline MD45 & CAR DLSVRGGMDV WG & IGHV3-53 & IYYC QQYGVSPEII & $\mathrm{FGO}$ & IGKV3-20 \\
\hline MD67 & CAR DLSVRGGMDV & IGHV3-53 & MYC QQFGSSPLT & FGG & IGKV3-20 \\
\hline MD62 & CAR DLQYYGMDV & IGHV3-53 & TYYC QQANSFPLT & FGG & IGKV1-12 \\
\hline MD47 & CAK DLVTAPSYEAFDI WG & IGHV3-23 & DYY QVWDSSSHH & VFGG & IGLV3-21 \\
\hline
\end{tabular}

d

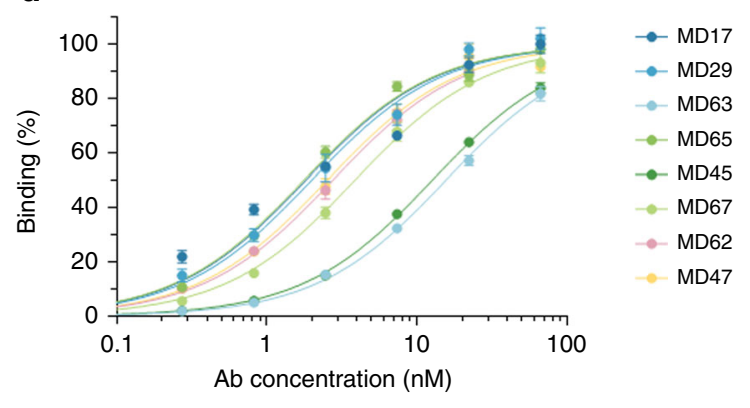

Germline
GKV1-39
GKV1-39
GKV1-39
GKV3-20
GKV3-20
GKV3-20
GKV1-12
GLV3-21

(2) e

\begin{tabular}{cccc}
\hline $\mathrm{Ab}$ & $K_{\text {on }}(1 / \mathrm{Ms})$ & $K_{\text {off }}(1 / \mathrm{s})$ & $K_{\mathrm{D}}(\mathrm{nM})$ \\
\hline $\mathrm{MD} 17$ & $1.0 \times 10^{5}$ & $2.6 \times 10^{-4}$ & 2.6 \\
$\mathrm{MD} 29$ & $3.7 \times 10^{5}$ & $1.4 \times 10^{-4}$ & 0.4 \\
$\mathrm{MD63}$ & $2.9 \times 10^{5}$ & $5.3 \times 10^{-4}$ & 3.4 \\
$\mathrm{MD65}$ & $3.1 \times 10^{5}$ & $5.8 \times 10^{-4}$ & 2.5 \\
$\mathrm{MD} 45$ & $2.0 \times 10^{5}$ & $5.5 \times 10^{-4}$ & 5.6 \\
$\mathrm{MD67}$ & $1.4 \times 10^{5}$ & $7.5 \times 10^{-4}$ & 5.8 \\
$\mathrm{MD62}$ & $2.3 \times 10^{5}$ & $6.3 \times 10^{-4}$ & 4.8 \\
$\mathrm{MD} 47$ & $1.8 \times 10^{5}$ & $0.9 \times 10^{-4}$ & 0.5
\end{tabular}

C

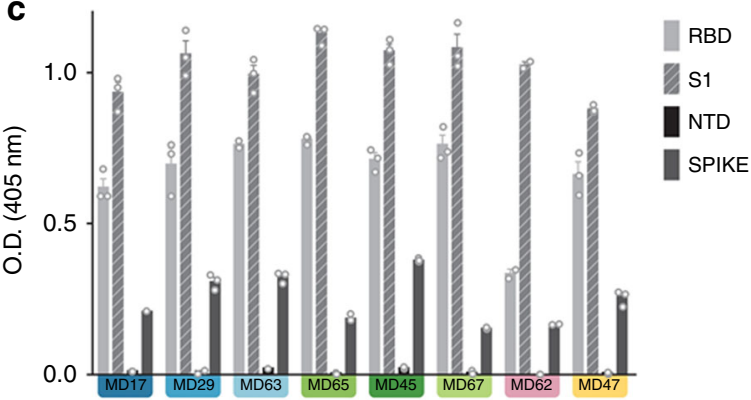


a

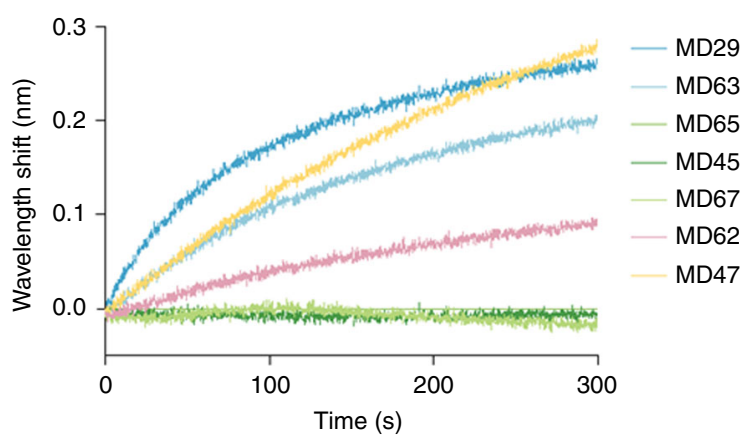

C

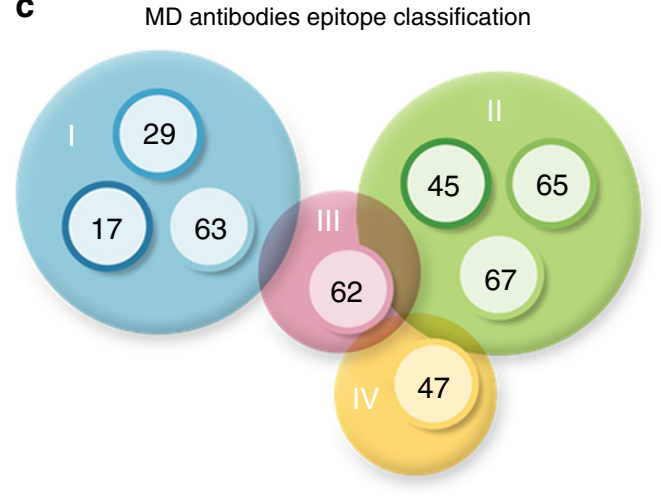

b

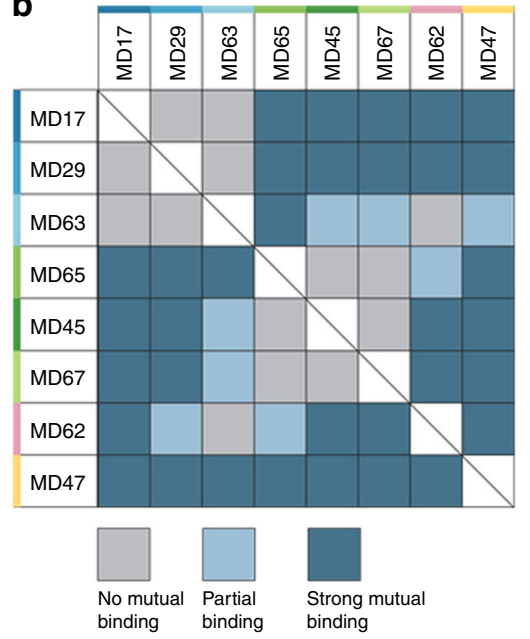

d

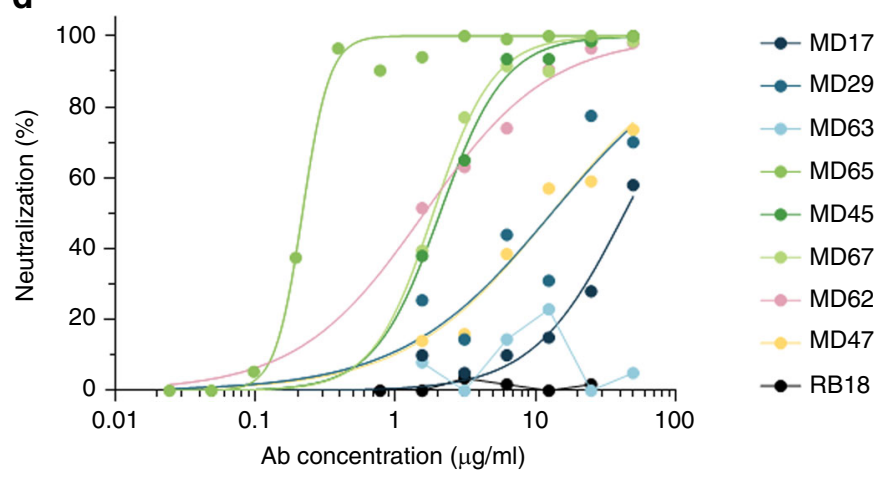

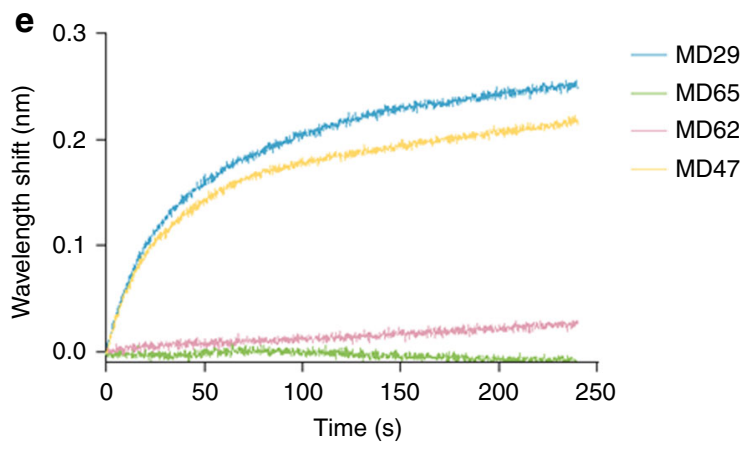

Fig. 2 Epitope binning and SARS-CoV-2 neutralization. a Biolayer interferometry (BLI) was applied for the epitope binning experiments. Representative assay results are shown for MD65 mAb. The purified antibody was biotinylated, immobilized on streptavidin sensor and saturated with RBD. The complex was then incubated for $300 \mathrm{~s}$ with each one of the indicated antibodies. Time 0 represents the binding to the MD65-RBD complex. $\mathbf{b}$ Complete epitope binning of the eight selected MD monoclonal antibodies. Binding was evaluated by the ability of each pair of antibodies to simultaneously bind RBD, using biolayer interferometry. The matrix presents the concluded epitope specificity on the basis of the various competition experiments; see Supplementary Fig. 3 for the detailed competition profiles obtained by the binning experiments. c Four noncompeting RBD binding epitopes were identified and accordingly classified into four groups: I (blue), II (green), III (pink) and IV (yellow). d SARS-CoV-2 in vitro neutralization using plaque reduction neutralization test (PRNT). Neutralization potency was determined by the ability of each antibody (at indicated concentrations) to reduce plaques formation; results are expressed as percent inhibition of control without Ab. The values represent average of duplicates. e Binding of human ACE2 to RBD in the presence of neutralizing antibodies (representing each of the epitope groups) was tested by BLI. Each of the biotinylated antibodies was immobilized on streptavidin sensor, saturated with RBD, washed and incubated with recombinant human ACE2 for 300 s. Time 0 represents the binding of the ACE2 to the antibodyRBD complex.

antibodies bind to the RBD epitope that spans the RBD residues 369-386, previously defined as the CR3022 epitope ${ }^{17}$.

In a previous study, a human anti-SARS-CoV-2 spike antibody (named S309) was shown to recognize a proteoglycan epitope ${ }^{25}$. This report prompted us to interrogate whether the ability of the novel antibodies described here to recognize and bind RBD also involves glycosylated moieties. To this objective, the binding ability of representative antibodies from each epitope-group was tested using an E.coli-expressed non-glycosylated recombinant version of RBD. The data demonstrate that glycosylation is not essential for recognition of RBD (Supplementary Fig. 6).

SARS-CoV-2 neutralization by the novel human antibodies. The neutralization potency of the antibodies was evaluated by 
plaque reduction neutralization test (PRNT) using VeroE6 cells infected with the pathogenic SARS-CoV-2. A fixed amount of the virus was incubated with increasing concentrations of each antibody, the mixture was then added to the cells, and the number of plaques was quantified $48 \mathrm{~h}$ later. Antibodies MD45, MD67, MD62, and MD65 displayed the highest neutralization potency, with a neutralization dose needed to inhibit $50 \%$ of the plaques $\left(\mathrm{NT}_{50}\right)$ of $2.1,1.9,1.6$, and $0.22 \mu \mathrm{g} / \mathrm{ml}$, respectively. MD65 exhibited the highest neutralization capacity amongst the entire set of antibodies (Fig. 2d). Interestingly, antibodies MD17, MD29, and MD63 shared DNA sequence homology indicative of a common germ line and competed for the same epitope, yet only the first two showed neutralizing activity $\left(\mathrm{NT}_{50}\right.$ of 43 and $13 \mu \mathrm{g} /$ $\mathrm{ml}$, respectively). This discrepancy may be explained by differences in the affinities of the antibodies in this group. A recent report similarly documented that antibody CR3022 (targeting an epitope overlapping with MD17 and MD29, see above) does not neutralize the novel SARS-CoV-2 presumably due to its low binding affinity to the RBD $(115 \mathrm{nM})^{17}$. Additional antibody tested in this study, MD47, which represents a unique epitopic group, also showed neutralizing activity with $\mathrm{NT}_{50}$ of $13 \mu \mathrm{g} / \mathrm{ml}$. Note that irrelevant $\mathrm{Ab}, \mathrm{RB} 18$ ( $\mathrm{scFv}-\mathrm{Fc}$ ) targeting abrin toxin, showed no neutralization against the virus.

One of the major mechanisms of antibody-mediated neutralization of SARS-CoV-1 and CoV-2 involves abrogation of the interaction between the viral spike protein and the human ACE2 receptor ${ }^{26}$. Therefore, in the current study, this aspect was further assessed by addressing the ability of representative antibodies from each epitope-group to prevent the binding of RBD to the huACE2 receptor by BLI analyses. Each antibody was immobilized on a BLI sensor, reacted with RBD and subsequently the ability of recombinant huACE2 to bind to the Ab-RBD complex was determined. The analysis established that MD29 and MD47 did not interfere with the binding of RBD to its receptor (Fig. 2e). This observation is in agreement with a previous report showing that antibody CR3022 (which recognizes the same epitope as antibody MD29) does not inhibit the RBD-ACE2 interaction in spite its ability to bind and neutralize SARS-CoV ${ }^{17}$. As opposed to MD29 and MD47, antibodies MD62 and MD65 completely inhibited the binding of RBD to ACE2. It is interesting to note that these two antibodies exhibited the highest neutralization potency (Fig. 2d), substantiating the essentiality of RBD-ACE2 prevention for efficient inhibition of viral activity.

To conclude, we report the isolation and characterization of a set of fully human, SARS-CoV-2 neutralizing antibodies that target four distinct epitopes on the spike RBD. As neutralizing antibodies are generally known to be useful as post-exposure therapy for viral infection and more specifically for treatment of human corona viral diseases ${ }^{3,27}$, we suggest that these antibodies might serve as an efficient treatment of COVID-19 patients or for prophylaxis immunization. Furthermore, since these neutralizing antibodies target different epitopes, they can be combined to further improve treatment efficacy and to reduce the risk of the emergence of treatment-escaping viral variants.

\footnotetext{
Methods

Blood samples. Sera and whole blood samples collected from five convalescent or severe COVID-19 patients were obtained under written inform consent and treated in accordance with the biosafety guidelines of the IIBR in BL3 facility. PBMCs were separated from fresh whole blood using density centrifugation by Ficoll. Sera samples were heat-inactivated $\left(20 \mathrm{~min}\right.$ at $\left.60^{\circ} \mathrm{C}\right)$ prior to use for binding or neutralizing assays. The study was approved by the Sheba Medical Center IRB Ethical Committee as well as by the Baruch Padeh Medical Center IRB Ethical Committee.
}

Phage display scFv libraries construction and phage Abs selection. Total RNA was purified from PBMCs using RNeasy mini kit (Qiagen GmbH, Germany). CDNA synthesis was performed using Verso cDNA synthesis kit
(Thermoscientific, USA) and used as a template for Abs variable region coding fragments amplification, as detailed in the Supplementary Data. Briefly, heavy and light Ig variable domains (VH and VL) were amplified, using specific primer set (Supplementary Table 1). The VH and VL used in PCR overlap extension reaction, resulted in $\mathrm{scFv}$ repertoire cloned into pCC16 phagemid vector ${ }^{28}$ using NcoI/NotI Total of $9.2 \times 10^{6}$ independent clones obtained, representing the library complexity.

For phage production, $25 \mathrm{ml}$ of logarithmic bacteria culture $\left(\mathrm{OD}_{600}=0.6\right)$ in YPD supplemented with $100 \mu \mathrm{g} / \mathrm{ml}$ ampicillin and $1 \%$ glucose (YPD-Amp-Glu) were infected with M13KO7 helper phage (New England Biolabs, USA) at $7 \times 10^{9}$ plaque-forming unit (PFU) per $\mathrm{ml}$ ( 1:20 multiplicity of infection) by incubating at $37^{\circ} \mathrm{C}$ for $30 \mathrm{~min}$ without shaking, followed by $30 \mathrm{~min}$ at $120 \mathrm{rpm}$. Infected cells were harvested by centrifugation $(1800 \times g$ for $5 \mathrm{~min})$ and resuspended in $100 \mathrm{ml}$ YPD supplemented with $100 \mu \mathrm{g} / \mathrm{ml}$ ampicillin and $50 \mu \mathrm{g} / \mathrm{ml}$ kanamycin. After overnight growth at $30^{\circ} \mathrm{C}$ at $200 \mathrm{rpm}$, the cells were removed by centrifugation $\left(1800 \times g\right.$ at $4^{\circ} \mathrm{C}$ for $\left.10 \mathrm{~min}\right)$. The culture supernatant containing the phages was filtered through a $0.45 \mu \mathrm{m}$ filter and then precipitated with $1 / 5$ volume of $20 \%$ PEG6000 (polyethylene glycol) in a $2.5 \mathrm{M} \mathrm{NaCl}$ solution, for $2 \mathrm{~h}$ on ice. Phage particles were pelleted by centrifugation $\left(9000 \times g\right.$ at $4{ }^{\circ} \mathrm{C}$ for $\left.1 \mathrm{~h}\right)$ and re-dissolved in $5 \mathrm{ml}$ Dulbecco's Phosphate Buffered Saline (PBS; Biological Industries, Israel).

Panning was performed against the recombinant huFc-RBD (prepared as described below) and $\mathrm{mFc}-\mathrm{S} 1$ (Sino Biological Inc., USA) directly absorbed to polystyrene plates (Maxisorb 96-well microtiter plates; Nunc, Sigma-Aldrich, USA) and against biotinylated-huFc-RBD (biotinylation performed using a commercial kit: EZ-Link sulfo-NHS-biotin, Pierce-Thermoscientific, USA) attached to streptavidin-coated magnetic beads (Dynabeads; Invitrogen, USA). All routine phage display techniques were performed essentially as described ${ }^{29}$. Blocking of plates, beads and phages was conducted for $60 \mathrm{~min}$ using two blocking solutions: $3 \%$ BSA (in PBS) or $2 \%$ skimmed milk and $0.05 \%$ Tween 20 in PBS. The blocking solutions were alternated between panning cycles. All washing steps were performed using PBST (PBS containing $0.05 \%$ Tween 20 and $2 \%$ BSA) or PBS. For each panning cycle, $5 \mu \mathrm{g} / \mathrm{ml}$ antigen was used to coat the polystyrene plate and after an overnight incubation, plates were washed and blocked. $20 \mu \mathrm{g}$ biotinylated-huFc RBD were incubated with $100 \mu \mathrm{l}$ streptavidin-coated magnetic beads for $30 \mathrm{~min}$, followed by blocking. For the first panning cycle, $\sim 1 \times 10^{11}$ phages were incubated with the antigen-coated plates for $60 \mathrm{~min}$ or with the blocked beads for $90 \mathrm{~min}$, followed by a total of three washes with PBST for the plates and four washing steps (x2 with PBST and $\mathrm{x} 2$ with PBS) for the beads. Phages were eluted by incubation with $1 \mathrm{ml}$ of $100 \mathrm{mM}$ Triethylamine (Sigma, Israel) for $30 \mathrm{~min}$ and following neutralization (in $200 \mu \mathrm{l} 1 \mathrm{M}$ Tris- $\mathrm{HCl}, \mathrm{pH} 7.4$ ), were used to infect $5 \mathrm{ml}$ of E.coli TG1 strain, by incubation at $37^{\circ} \mathrm{C}$ for $30 \mathrm{~min}$ without shaking followed by $30 \mathrm{~min}$ at $120 \mathrm{rpm}$. The bacterial culture was plated on YPD-Amp-Glu agar, and incubated overnight at $30^{\circ} \mathrm{C}$. Clones were harvested into $5 \mathrm{ml}$ YPD-Amp-Glu with $20 \%$ glycerol solution and phage production for the next round of panning was conducted in $10 \mathrm{ml}$ medium, as described above. Two additional panning cycles were conducted essentially similarly, with the following modifications: $10^{10}$ and $10^{9}$ phages were used as input (for the 2 nd and 3rd cycles, respectively) and the washing steps were increased to include six washes of PBST for the antigen-coated plates and 10 washes (x8 PBST and x2 PBS) for the beads.

Single colonies were randomly picked from the third cycle output and screen of specific binders was performed, using phage ELISA against huFc-RBD Vs huFcNTD.

Single-chain Fv (scFv) individual clone diversity and sequence verification TAB-RI_For (CCATGATTACGCCAAGCTTTGGAGCC) and CBD-AS_Rev (GAATTCAACCTTCAAATTGCC) phagemid specific primers were used for colony PCR and sequence analysis of scFv Ab individual clones (Supplementary Fig. 1). Colony PCR products, were analyzed on $1.5 \%$ agarose gel, to confirm the intact of the scFv. Restriction fragment size polymorphism (RFLP), was performed using MvaI (FastDigest \#FD0554; Thermoscientific, USA) to evaluate sequence variability of $s c F v$ individual clones. Following colony PCR, $5 \mu \mathrm{l}$ of the PCR products were taken directly for restriction with $0.5 \mu \mathrm{l}$ MvaI and $1 \mu \mathrm{l}$ buffer x10 (provided by the manufacturer) in a $10 \mu \mathrm{l}$ reaction volume. Restriction was conducted for $1 \mathrm{~h}$ at $37^{\circ} \mathrm{C}$, and the entire reaction mix was then resolved on $3 \%$ agarose gel. Nucleic acid sequence analysis of individual $s c F v$ fragments, was performed to the colony PCR product, using SeqStudio Genetic Analyzer (Applied Biosystems, USA).

Expression of SARS-CoV-2 spike recombinant protein. Mammalian cell codonoptimized nucleic sequence, coding for SARS-CoV-2 spike glycoprotein (GenPept QHD43416 ORF [https://www.ncbi.nlm.nih.gov/protein/1791269090]), was used to design pcDNA3.1+ based expression plasmids, mediating recombinant expression of the entire spike glycoprotein (amino acids 1-1207), receptor-binding domain (RBD; amino acids 1-15 and 318-542), N-terminal domain (NTD; amino acids 1-305) and S1 (amino acids 1-685). A stabilized soluble version of the spike protein was designed by inclusion of the proline substitutions at positions 986 and 987, and disruptive replacement of the furin cleavage site RRAR (residues at position 682-685) with GSAS, as reported ${ }^{30,31}$. C-terminal his-tag as well as streptag, was included in all constructs in order to facilitate protein purification. Expression of the recombinant proteins was performed using ExpiCHO ${ }^{\mathrm{TM}}$ 
Expression system (Thermoscientific, USA) following purification using His$\operatorname{Trap}^{\mathrm{TM}}$ (GE Healthcare, UK) and Strep-Tactin ${ }^{\circ} \mathrm{XT}$ (IBA, Germany).

In addition, huFc-RBD fused protein was expressed using previously designed Fc-fused protein expression vector ${ }^{32}$, giving rise to a protein comprising of two RBD moieties (amino acids 318-542, see accession number of the $\mathrm{S}$ protein above) owing to the homodimeric human (gammal)Fc domain (huFc). Expression of the recombinant proteins performed using ExpiCHO ${ }^{\mathrm{TM}}$ Expression system (Thermoscientific, USA) following purification using HiTrap Protein-A column (GE healthcare, UK). All purified proteins preserved in PBS.

Production of scFv-Fc antibodies. Phagemid DNA of the desired clones were isolated using QIAprep spin Miniprep kit (Qiagen, GmbH, Hilden, Germany), and the entire scFv was cloned into a pcDNA3.1+ based expression vector that was modified, providing the scFv with the human (IgG1) $\mathrm{CH} 2-\mathrm{CH} 3 \mathrm{Fc}$ fragments, resulting in scFv-Fc antibody format. ScFv-Fc were expressed using ExpiCHO ${ }^{\mathrm{TM}}$ Expression system (Thermoscientific, USA, Cat\# A29133) and purified on HiTrap Protein-A column (GE healthcare, UK). The integrity and purity of the $\mathrm{scFv}-\mathrm{Fc}$ Abs were analyzed using SDS-PAGE (Supplementary Fig. 7).

ELISA. Direct ELISA ${ }^{33}$ consisted of coating microtiter plates with $2 \mu \mathrm{g} / \mathrm{ml}$ of recombinant SARS-CoV-2 spike, S1 domain, RBD or NTD subunits. For phage ELISA, HRP-conjugated anti-M13 antibody (Sino Biological, USA, Cat\# 11973MM05T-H lot HO13AU601; used at 1:5000 working dilution) was used following detection with TMB substrate (Millipore, USA). ELISA of both sera and recombinant scFv-Fc human antibodies was applied with AP-conjugated Donkey antihuman IgG (Jackson ImmunoResearch, USA, Cat\# 709-055-149 lot 130049; used at 1:2000 working dilution) following detection using PNPP substrate (Sigma, Israel).

\section{Biolayer interferometry for affinity measurements and epitope binning.}

Binding studies were carried out using the Octet system (ForteBio, USA, Version $8.1,2015)$ that measures biolayer interferometry (BLI). All steps were performed at $30^{\circ} \mathrm{C}$ with shaking at $1500 \mathrm{rpm}$ in a black 96-well plate containing $200 \mu \mathrm{l}$ solution in each well. Streptavidin-coated biosensors were loaded with biotinylated scFv-Fc antibody $(1-5 \mu \mathrm{g} / \mathrm{ml})$ to reach $0.7-1 \mathrm{~nm}$ wavelength shift followed by a wash. The sensors were then reacted for $300 \mathrm{~s}$ with increasing concentrations of monomeric RBD (association phase) and then transferred to buffer-containing wells for another $600 \mathrm{~s}$ (dissociation phase). Binding and dissociation were measured as changes over time in light interference after subtraction of parallel measurements from unloaded biosensors. Sensorgrams were fitted with a 1:1 binding model (Supplementary Fig. 2) using the Octet data analysis software 8.1 (Fortebio, USA, 2015), and the presented values are an average of several repeated measurements. For the binning experiments of antibodies pairs, antibody-loaded sensors were incubated with a fixed RBD concentration $(300 \mathrm{nM})$, washed and incubated with the non-labeled antibody counterpart. In each set of experiments, the background signal was obtained from a parallel sensor incubated with the homologous antibody and sensograms are presented after subtraction of the background signal (Supplementary Fig. 3). In addition, binning experiments were performed in the absence of RBD, in order to validate the absence of non-specific binding (Supplementary Fig. 4). To study the binding of ACE2 to RBD in the presence of antibodies, antibody-loaded sensors were incubated with a RBD, washed and incubated with huACE $(10 \mu \mathrm{g} / \mathrm{ml}$; Sino Biological Inc., USA).

Cells. Vero E6 (ATCC ${ }^{\circledR}$ CRL-1586 ${ }^{\mathrm{TM}}$ ) were obtained from the American Type Culture Collection (Summit Pharmaceuticals International, Japan). Cells were used and maintained in Dulbecco's modified Eagle's medium (DMEM) supplemented with $10 \%$ fetal bovine serum (FBS), MEM non-essential amino acids, $2 \mathrm{nM} \mathrm{L-}$ Glutamine, 100 Units $/ \mathrm{ml}$ Penicilin, $0.1 \mathrm{mg} / \mathrm{ml}$ streptomycin and 12.5 Units $/ \mathrm{ml}$ Nystatin (Biological Industries, Israel). Cells were cultured at $37^{\circ} \mathrm{C}, 5 \% \mathrm{CO}_{2}$ at $95 \%$ air atmosphere.

ExpiCHO-S (Thermoscientific, USA, Cat\# A29127) were used for expression of recombinant proteins as described above.

Plaque reduction neutralization test (PRNT). SARS-CoV-2 (GISAID accession EPI_ISL_406862) was kindly provided by Bundeswehr Institute of Microbiology, Munich, Germany. Stocks were prepared by infection of Vero E6 cells for two days. When viral cytopathic effect (CPE) was observed, media were collected, clarified by centrifugation, aliquoted and stored at $-80^{\circ} \mathrm{C}$. Titer of stock was determined by plaque assay using Vero E6 cells. Handling and working with SARS-CoV-2 was conducted in BL3 facility in accordance with the biosafety guidelines of the IIBR.

For plaque reduction neutralization test $(\mathrm{PRNT})^{34}$, Vero E6 cells were seeded overnight (as detailed above) at a density of $0.5 \times 10^{6}$ cells/well in 12-well plates. Antibody samples were 2-fold serially diluted (ranging from 100 to $0.1 \mu \mathrm{g} / \mathrm{ml}$ ) in $400 \mu \mathrm{l}$ of MEM supplemented with $2 \%$ FBS, MEM non-essential amino acids, $2 \mathrm{nM}$ L-Glutamine, 100 Units $/ \mathrm{ml}$ Penicilin, $0.1 \mathrm{mg} / \mathrm{ml}$ streptomycin and $12.5 \mathrm{Units} / \mathrm{ml}$ Nystatin (Biological Industries, Israel). $400 \mu \mathrm{l}$ containing $300 \mathrm{PFU} / \mathrm{ml}$ of SARS$\mathrm{CoV}-2$ virus were then added to the mAb solution supplemented with $0.25 \%$ guinea pig complement sera (Sigma, Israel) and the mixture incubated at $37{ }^{\circ} \mathrm{C}, 5 \%$ $\mathrm{CO}_{2}$ for $1 \mathrm{~h}$. Monolayers were then washed once with DMEM w/o FBS and $200 \mu \mathrm{l}$ of each mAb-virus mixture was added in duplicates to the cells for $1 \mathrm{~h}$. Virus mixture w/o mAb was used as control. $2 \mathrm{ml}$ overlay [MEM containing $2 \%$ FBS and $0.4 \%$ tragacanth (Sigma, Israel)] were added to each well and plates were further incubated at $37^{\circ} \mathrm{C}, 5 \% \mathrm{CO}_{2}$ for $48 \mathrm{~h}$. The number of plaques in each well was determined following media aspiration, cells fixation and staining with $1 \mathrm{ml}$ of crystal violet (Biological Industries, Israel). $\mathrm{NT}_{50}$ was defined as $\mathrm{mAb}$ concentration at which the plaque number was reduced by $50 \%$, compared to plaque number of the control (in the absence of $\mathrm{Ab}$ ).

Reporting summary. Further information on research design is available in the Nature Research Reporting Summary linked to this article.

\section{Data availability}

Antibodies are available (by contacting Ohad Mazor from the Israel Institute for Biological Research; ohadm@iibr.gov.il) for research purposes only under an MTA, which allows the use of the antibodies for non-commercial purposes but not their disclosure to third parties. All other data are available from the corresponding authors upon reasonable requests. Source data are provided with this paper.

Received: 20 May 2020; Accepted: 5 August 2020;

Published online: 27 August 2020

\section{References}

1. https://www.who.int/health-topics/coronavirus\#tab=tab_1.

2. Iwasaki, A. \& Yang, Y. The potential danger of suboptimal antibody responses in COVID-19. Nat Rev Immunol, https://doi.org/10.1038/s41577-020-0321-6 (2020).

3. Prabakaran, P. et al. Potent human monoclonal antibodies against SARS CoV, Nipah and Hendra viruses. Expert Opin. Biol. Ther. 9, 355-368 (2009).

4. Zhou, H. et al. Structural definition of a neutralization epitope on the Nterminal domain of MERS-CoV spike glycoprotein. Nat. Commun. 10, 3068 (2019).

5. Ying, T. et al. Exceptionally potent neutralization of Middle East respiratory syndrome coronavirus by human monoclonal antibodies. J. Virol. 88, 7796-7805 (2014)

6. Saphire, E. O., Schendel, S. L., Gunn, B. M., Milligan, J. C. \& Alter, G. Antibody-mediated protection against Ebola virus. Nat. Immunol. 19, 1169-1178 (2018).

7. Hoffmann, M., Kleine-Weber, H. \& Pohlmann, S. A multibasic cleavage site in the spike protein of SARS-CoV-2 is essential for infection of human lung cells. Mol. Cell 78, 779-784 e775 (2020).

8. Walls, A. C. et al. Structure, function, and antigenicity of the SARS-CoV-2 spike glycoprotein. Cell 181, 281-292 e286 (2020).

9. Hoffmann, M. et al. SARS-CoV-2 cell entry depends on ACE2 and TMPRSS2 and is blocked by a clinically proven protease inhibitor. Cell 181, 271-280 e278 (2020).

10. Yu, X. et al. Structural basis for the neutralization of MERS-CoV by a human monoclonal antibody MERS-27. Sci. Rep. 5, 13133 (2015).

11. Rockx, B. et al. Escape from human monoclonal antibody neutralization affects in vitro and in vivo fitness of severe acute respiratory syndrome coronavirus. J. Infect. Dis. 201, 946-955 (2010).

12. Rockx, B. et al. Structural basis for potent cross-neutralizing human monoclonal antibody protection against lethal human and zoonotic severe acute respiratory syndrome coronavirus challenge. J. Virol. 82, 3220-3235 (2008).

13. Reguera, J. et al. Structural bases of coronavirus attachment to host aminopeptidase $\mathrm{N}$ and its inhibition by neutralizing antibodies. PLoS Pathog. 8, e1002859 (2012).

14. Hwang, W. C. et al. Structural basis of neutralization by a human anti-severe acute respiratory syndrome spike protein antibody, 80R. J. Biol. Chem. 281, 34610-34616 (2006)

15. Tian, X. et al. Potent binding of 2019 novel coronavirus spike protein by a SARS coronavirus-specific human monoclonal antibody. Emerg. Microbes Infect. 9, 382-385 (2020).

16. Wang, C. et al. A human monoclonal antibody blocking SARS-CoV-2 infection. Nat. Commun. 11, 2251 (2020).

17. Yuan, M. et al. A highly conserved cryptic epitope in the receptor binding domains of SARS-CoV-2 and SARS-CoV. Science 368, 630-633 (2020).

18. $\mathrm{Wu}, \mathrm{Y}$. et al. A noncompeting pair of human neutralizing antibodies block COVID-19 virus binding to its receptor ACE2. Science, https://doi.org/ 10.1126/science.abc2241 (2020)

19. Yoo, D. \& Deregt, D. A single amino acid change within antigenic domain II of the spike protein of bovine coronavirus confers resistance to virus neutralization. Clin. Diagn. Lab Immunol. 8, 297-302 (2001). 
20. Ye, J., Ma, N., Madden, T. L. \& Ostell, J. M. IgBLAST: an immunoglobulin variable domain sequence analysis tool. Nucleic Acids Res 41, W34-W40 (2013).

21. Mechaly, A., Cohen, H., Cohen, O. \& Mazor, O. A biolayer interferometrybased assay for rapid and highly sensitive detection of biowarfare agents. Anal. Biochem 506, 22-27 (2016).

22. Abdiche, Y. N., Malashock, D. S., Pinkerton, A. \& Pons, J. Exploring blocking assays using Octet, ProteOn, and Biacore biosensors. Anal. Biochem 386, 172-180 (2009).

23. Abdiche, Y. N. et al. High-throughput epitope binning assays on label-free array-based biosensors can yield exquisite epitope discrimination that facilitates the selection of monoclonal antibodies with functional activity. PLoS One 9, e92451 (2014).

24. Noy-Porat, T. et al. Extended therapeutic window for post-exposure treatment of ricin intoxication conferred by the use of high-affinity antibodies. Toxicon 127, 100-105 (2017).

25. Pinto, D. et al. Cross-neutralization of SARS-CoV-2 by a human monoclonal SARS-CoV antibody. Nature, https://doi.org/10.1038/s41586-020-2349-y (2020).

26. Cao, Y. et al. Potent neutralizing antibodies against SARS-CoV-2 identified by high-throughput single-cell sequencing of convalescent patients' B cells. Cell, https://doi.org/10.1016/j.cell.2020.05.025 (2020).

27. Jiang, S., Hillyer, C. \& Du, L. Neutralizing Antibodies against SARS-CoV-2 and Other Human Coronaviruses: (Trends in Immunology 41, 355-359; 2020). Trends Immunol, https://doi.org/10.1016/j.it.2020.04.008 (2020).

28. Azriel-Rosenfeld, R., Valensi, M. \& Benhar, I. A human synthetic combinatorial library of arrayable single-chain antibodies based on shuffling in vivo formed CDRs into general framework regions. J. Mol. Biol. 335 177-192 (2004).

29. Benhar, I. \& Reiter, Y. Phage display of single-chain antibody constructs. Curr. Protoc. Immunol. 48, 10.19B.1-10.19B.31 (2002).

30. Wrapp, D. et al. Cryo-EM structure of the $2019-\mathrm{nCoV}$ spike in the prefusion conformation. Science 367, 1260-1263 (2020).

31. Stadlbauer, D. et al. SARS-CoV-2 seroconversion in humans: a detailed protocol for a serological assay, antigen production, and test setup. Curr. Protoc. Microbiol 57, e100 (2020).

32. Noy-Porat, T. et al. Acetylcholinesterase-Fc fusion protein (AChE-Fc): a novel potential organophosphate bioscavenger with extended plasma half-life. Bioconjug Chem. 26, 1753-1758 (2015).

33. Noy-Porat, T. et al. Isolation of anti-ricin protective antibodies exhibiting high affinity from immunized non-human primates. Toxins $\mathbf{8}$, https://doi.org/ 10.3390/toxins8030064 (2016).

34. Eyal, O. et al. Development of a tissue-culture-based enzyme-immunoassay method for the quantitation of anti-vaccinia-neutralizing antibodies in human sera. J. Virol. Methods 130, 15-21 (2005).

\section{Acknowledgements}

We thank Dr. Itzchak Levy and Dr. Asaf Biber from the Sheba Medical Center and Dr. Hagar Mizrahi, Dr. Hiba Zayyad and Dr. Moshe Matan from the Baruch Padeh Medical
Center, for kindly providing the blood samples collected from the COVID-19 patients. We thank Dr. Alon Ben-David for providing E.coli-expressed RBD. We wish to express our gratitude to our colleagues Dr. Ofer Cohen, Dr. Emanuelle Mamroud, Moshe Mantzur, Moshe Aftalion, Noa Caspi, Yentl Evgy, Hila Cohen, Dr. Liat Bar-On, Dr. Ronit Aloni-Grinstein, David Gur, Sarit Sterenberg and Tamar Aminov for fruitful discussions and support. We acknowledge the IIBR administrative personnel for their commitment to the project and Dalit Brener for graphical design.

\section{Author contributions}

R.R., O.M., T.N.-P. and E.M. conceived and designed the experiments and wrote the manuscript. T.N.-P., E.M., R.A., A.M., Y.L., A.B.-K., A.Z., H.T., Y.Y.-R., M.I., E.E., H.A., S.M., T.C., S.W., E.P., O.R., N.P., T.I., O.M. and R.R. designed and performed the experiments and analyzed the data. S.Y. and S.S added fruitful discussions. R.R. and O.M. supervised the project.

\section{Competing interests}

Patent application for the described antibodies was filed by the Israel Institute for Biological Research. None of the authors declared any additional competing interests.

\section{Additional information}

Supplementary information is available for this paper at https://doi.org/10.1038/s41467 020-18159-4.

Correspondence and requests for materials should be addressed to O.M. or R.R.

Peer review information Nature Communications thanks Markus Seeger, and the other, anonymous, reviewer for their contribution to the peer review of this work.

Reprints and permission information is available at http://www.nature.com/reprints

Publisher's note Springer Nature remains neutral with regard to jurisdictional claims in published maps and institutional affiliations.

Open Access This article is licensed under a Creative Commons Attribution 4.0 International License, which permits use, sharing, adaptation, distribution and reproduction in any medium or format, as long as you give appropriate credit to the original author(s) and the source, provide a link to the Creative Commons license, and indicate if changes were made. The images or other third party material in this article are included in the article's Creative Commons license, unless indicated otherwise in a credit line to the material. If material is not included in the article's Creative Commons license and your intended use is not permitted by statutory regulation or exceeds the permitted use, you will need to obtain permission directly from the copyright holder. To view a copy of this license, visit http://creativecommons.org/ licenses/by/4.0/.

(C) The Author(s) 2020 